\title{
XYZ - spectra from QCD Laplace Sum Rules at Higher Orders th
}

\author{
R. Albuquerque \\ Faculty of Technology, Rio de Janeiro State University (FAT,UERJ), Brazil \\ S. Narison* \\ Laboratoire Univers et Particules de Montpellier (LUPM), CNRS-IN2P3, \\ Case 070, Place Eugène Bataillon, 34095 - Montpellier, France. \\ A. Rabemananjara, D. Rabetiarivony ${ }^{1}$, G. Randriamanatrika ${ }^{1}$ \\ Institute of High-Energy Physics of Madagascar (iHEPMAD) \\ University of Ankatso, Antananarivo 101, Madagascar
}

\begin{abstract}
We review our results in Refs. [1, 2] for the masses and couplings of heavy-light $\bar{D} D(\bar{B} B)$-like molecules and $(\overline{Q q})(Q q)$-like four-quark states from relativistic QCD Laplace sum rules (LSR) where next-to-next-to-leading order (N2LO) PT corrections in the chiral limit, next-to-leading order (NLO) SU3 PT corrections and non-perturbative contributions up to dimension $d=6-8$ are included. The factorization properties of molecule and four-quark currents have been used for the estimate of the higher order PT corrections. New integrated compact expressions of the spectral functions at leading order (LO) of perturbative QCD and up to dimensions $d \leq(6-8)$ non-perturbative condensates are presented. The results are summarized in Tables 5 to 10 , from which we conclude, within the errors, that the observed $\mathrm{XZ}$ states are good candidates for being $1^{++}$and $0^{++}$molecules or/and four-quark states, contrary to the observed Y states which are too light compared to the predicted $1^{- \pm}$and $0^{- \pm}$states. We find that the SU3 breakings are relatively small for the masses $(\leq 10$ (resp. 3) \%) for the charm (resp. bottom) channels while they are large $(\leq 20 \%)$ for the couplings which decrease faster $\left(1 / m_{b}^{3 / 2}\right)$ than $1 / m_{b}^{1 / 2}$ of HQET. QCD spectral sum rules (QSSR) approach cannot clearly separate (within the errors) molecules from four-quark states having the same quantum numbers. Results for the $\bar{B} K(\bar{D} K)$-like molecules and $(\overline{Q q})(u s)$-like four-quark states from [3] are also reviewed which do not favour the molecule or/and four-quark interpretation of the $X(5568)$. We suggest to scan the charm $(2327 \sim 2444) \mathrm{MeV}$ and bottom $(5173 \sim 5226) \mathrm{MeV}$ regions for detecting the (unmixed) $(\overline{c u}) d s$ and $(\overline{b u}) d s$ via eventually their radiative or $\pi+$ hadrons decays and reconsider more carefully the properties of the eventual $D_{s 0}^{*}(2317)$ candidate. We expect that future experimental data and lattice results will check our predictions.
\end{abstract}

Keywords: QCD Spectral Sum Rules, Perturbative and Non-perturbative QCD, Exotic hadrons, Masses and Decay constants.

\footnotetext{
Talk presented at QCD18 (2-6 july 2018, Montpellier-FR) and HEPMAD18 (6-11 September 2018, Antananarivo-MG) ${ }^{*}$ ICTP-Trieste high-energy physics consultant for Madagascar Email addresses: raphael. albuquerque@uerj.br (R.
}

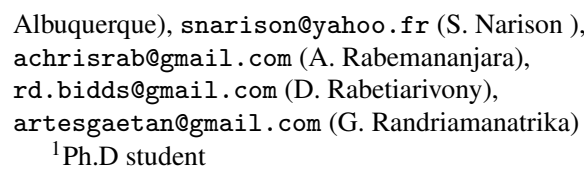

Albuquerque), snarison@yahoo.fr (S. Narison ), achrisrab@gmail.com (A. Rabemananjara), rd.bidds@gmail.com (D. Rabetiarivony), artesgaetan@gmail.com (G. Randriamanatrika)

${ }^{1} \mathrm{Ph} . \mathrm{D}$ student 


\section{Introduction}

A large amount of exotic hadrons which differ from the standard $\bar{c} c$ charmonium and $\bar{b} b$ bottomium radial excitation states have been recently discovered in Bfactories and have stimulated different theoretical interpretations. Most of them have been assigned as fourquark and/or molecule states. In this paper, we use relativistic Laplace sum rules $[4,-8]^{2}$ to improve some previous LO results for the masses and decay constants of the XYZ exotic heavy-light mesons obtained in the chiral limit [13-15]. In so doing, we include N2LO PT corrections to the heavy light correlators. We pursue our investigation by adding the SU3 NLO PT corrections. With these higher order (HO) PT contributions, we add the LO contribution of condensates up to dimension six. We do not include into the analysis contributions of condensates of higher dimension $(d \geq 8)$ but only consider their effects as a source of systematic errors due to the truncation of the Operator Product Expansion (OPE). This work is a part of the original papers in [1-3, 16, 17].

\section{Molecules and four-quark two point functions}

We shall work with the transverse part $\Pi_{m o l}^{(1)}$ of the two-point spectral functions 3

$$
\begin{aligned}
\Pi_{m o l}^{\mu v}(q) & \equiv i \int d^{4} x e^{i q \cdot x}\left\langle 0\left|T\left[O_{m o l}^{\mu}(x) O_{m o l}^{v \dagger}(0)\right]\right| 0\right\rangle \\
& =-\Pi_{m o l}^{(1)}\left(q^{2}\right)\left(g^{\mu v}-\frac{q^{\mu} q^{v}}{q^{2}}\right)+\Pi_{m o l}^{(0)}\left(q^{2}\right) \frac{q^{\mu} q^{v}}{q^{2}},(1)
\end{aligned}
$$

for the spin 1 states while for the spin 0 ones, we shall use the two-point functions $\psi_{m o l}\left(q^{2}\right)$ built directly from the (pseudo)scalar currents:

$$
\psi_{m o l}\left(q^{2}\right)=i \int d^{4} x e^{i q \cdot x}\left\langle 0\left|T\left[O_{m o l}(x) O_{m o l}(0)\right]\right| 0\right\rangle,
$$

which is related to $\Pi^{(0)}$ appearing in Eq.1 via Ward identities $[9,10,18]$. Thanks to their analyticity properties $\Pi_{m o l}^{(1,0)}$ and $\psi_{m o l}$ obey the dispersion relation:

$$
\Pi_{m o l}^{(1,0)}\left(q^{2}\right) ; \psi_{m o l}\left(q^{2}\right)=\frac{1}{\pi} \int_{4 M_{Q}^{2}}^{\infty} d t \frac{\operatorname{Im} \Pi_{m o l}^{(1,0)}(t) ; \operatorname{Im} \psi_{m o l}(t)}{t-q^{2}-i \epsilon}
$$

where $\operatorname{Im} \Pi_{m o l}^{(1,0)}(t)$ and $\operatorname{Im} \psi_{m o l}(t)$ are the spectral functions.

\footnotetext{
${ }^{2}$ For reviews, see [9. 12]

${ }^{3}$ Hereafter, similar expressions will be obtained for the four-quark states by replacing the sub-index $\mathrm{mol}$ by $4 q$.
}

\section{- Interpolating currents}

The interpolating currents $O_{m o l}$ and $O_{4 q}$ respectively for the $\bar{D} D(\bar{B} B)$-like molecules and four-quark states are given in Tables 1 and 2 The ones describing $\bar{B} K(\bar{D} K)$ (resp. $(\overline{Q q})(u s)$ )-like molecules (resp. four-quark) states are presented in Table 3

Table 1: Interpolating currents $O_{m o l}$ with a definite C-parity describing the molecule-like states. $M \equiv D$ (resp. $B$ ) and $Q \equiv c$ (resp. $b$ ) for the $\bar{D} D($ resp. $\bar{B} B)$-like molecules. $q \equiv d($ resp. $s)$ for the chiral limit (resp. SU3 breaking).

\begin{tabular}{lll}
\hline \hline States & $J^{P C}$ & Molecule currents $O_{m o l}(x)$ \\
\hline Scalar & & \\
$\bar{M} M, \bar{M}_{s} M_{s}$ & $0^{++}$ & $\left(\bar{q} \gamma_{5} Q\right)\left(\bar{Q} \gamma_{5} q\right)$ \\
$\bar{M}^{*} M^{*}, \bar{M}_{s}^{*} M_{s}^{*}$ & & $\left(\bar{q} \gamma_{\mu} Q\right)\left(\bar{Q} \gamma^{\mu} q\right)$ \\
$\bar{M}_{0}^{*} M_{0}^{*}, \bar{M}_{s 0}^{*} M_{s 0}^{*}$ & & $(\bar{q} Q)(\bar{Q} q)$ \\
$\bar{M}_{1} M_{1}, \bar{M}_{s 1} M_{s 1}$ & & $\left(\bar{q} \gamma_{\mu} \gamma_{5} Q\right)\left(\bar{Q} \gamma^{\mu} \gamma_{5} q\right)$ \\
Axial-vector & $1^{++}$ & $\frac{i}{\sqrt{2}}\left[\left(\bar{Q} \gamma_{\mu} q\right)\left(\bar{q} \gamma_{5} Q\right)-\left(\bar{q} \gamma_{\mu} Q\right)\left(\bar{Q} \gamma_{5} q\right)\right]$ \\
$\bar{M}^{*} M, \bar{M}_{s}^{*} M_{s}$ & & $\frac{i}{\sqrt{2}}\left[(\bar{q} Q)\left(\bar{Q} \gamma_{\mu} \gamma_{5} q\right)+(\bar{Q} q)\left(\bar{q} \gamma_{\mu} \gamma_{5} Q\right)\right]$ \\
$\bar{M}_{0}^{*} M_{1}, \bar{M}_{s 0}^{*} M_{s 1}$ & & $\frac{1}{\sqrt{2}}\left[(\bar{q} Q)\left(\bar{Q} \gamma_{5} q\right) \pm(\bar{Q} q)\left(\bar{q} \gamma_{5} Q\right)\right]$ \\
Pseudoscalar & $0^{- \pm}$ & $\frac{1}{\sqrt{2}}\left[\left(\bar{Q} \gamma_{\mu} q\right)\left(\bar{q} \gamma^{\mu} \gamma_{5} Q\right) \mp\left(\bar{Q} \gamma_{\mu} \gamma_{5} q\right)\left(\bar{q} \gamma^{\mu} Q\right)\right]$ \\
$M_{0}^{*} M, M_{s 0}^{*} M_{s}$ & & $\frac{1}{\sqrt{2}}\left[(\bar{q} Q)\left(\bar{Q} \gamma_{\mu} q\right) \mp(\bar{Q} q)\left(\bar{q} \gamma_{\mu} Q\right)\right]$ \\
$M^{*} M_{1}, M_{s}^{*} M_{s 1}$ & & $\frac{1}{\sqrt{2}}\left[\left(\bar{Q} \gamma_{\mu} \gamma_{5} q\right)\left(\bar{q} \gamma_{5} Q\right) \pm\left(\bar{q} \gamma_{\mu} \gamma_{5} Q\right)\left(\bar{Q} \gamma_{5} q\right)\right]$ \\
Vector & $1^{- \pm}$ & \\
$M_{0}^{*} M^{*}, M_{s 0}^{*} M_{s}^{*}$ & & \\
$M M_{1}, M_{s} M_{s 1}$ & & \\
\hline \hline
\end{tabular}

Table 2: Interpolating currents describing the four-quark states. $Q \equiv c$ (resp. $b$ ) and $q \equiv d($ resp. $s$ ). $k$ is an arbitrary current mixing where the optimal value is found to be $k=0$ from [14]

\begin{tabular}{lll|l}
\hline \hline States & $J^{P}$ & Four-quark currents $O_{4 q}(x)$ \\
\hline Scalar & $0^{+}$ & $\epsilon_{a b c} \epsilon_{d e c}$ & $\left.\left(q_{a}^{T} C \gamma_{5} Q_{b}\right)\left(\bar{q}_{d} \gamma_{5} C \bar{Q}_{e}^{T}\right)+k\left(q_{a}^{T} C Q_{b}\right)\left(\bar{q}_{d} C \bar{Q}_{e}^{T}\right)\right]$ \\
Axial-vector & $1^{+}$ & $\epsilon_{a b c} \epsilon_{d e c}$ & $\left.\left(q_{a}^{T} C \gamma_{5} Q_{b}\right)\left(\bar{q}_{d} \gamma_{\mu} C \bar{Q}_{e}^{T}\right)+k\left(q_{a}^{T} C Q_{b}\right)\left(\bar{q}_{d} \gamma_{\mu} \gamma_{5} C \bar{Q}_{e}^{T}\right)\right]$ \\
Pseudoscalar & $0^{-}$ & $\epsilon_{a b c} \epsilon_{d e c}$ & $\left.\left(q_{a}^{T} C \gamma_{5} Q_{b}\right)\left(\bar{q}_{d} C \bar{Q}_{e}^{T}\right)+k\left(q_{a}^{T} C Q_{b}\right)\left(\bar{q}_{d} \gamma_{5} C \bar{Q}_{e}^{T}\right)\right]$ \\
Vector & $1^{-}$ & $\epsilon_{a b c} \epsilon_{d e c}$ & $\left.\left(q_{a}^{T} C \gamma_{5} Q_{b}\right)\left(\bar{q}_{d} \gamma_{\mu} \gamma_{5} C \bar{Q}_{e}^{T}\right)+k\left(q_{a}^{T} C Q_{b}\right)\left(\bar{q}_{d} \gamma_{\mu} C \bar{Q}_{e}^{T}\right)\right]$ \\
\hline \hline
\end{tabular}

Table 3: Interpolating currents describing the $X(5568)$-like states. $M \equiv D($ resp. $B)$ and $Q \equiv c($ resp. $b)$.

\begin{tabular}{lll}
\hline \hline Nature & $J^{P}$ & Current \\
& & \\
\hline Molecule & & $\left(\bar{Q} i \gamma_{5} u\right)\left(\bar{d} i \gamma_{5} s\right)$ \\
$\bar{M} K$ & $0^{+}$ & $\left(\bar{Q} i \gamma_{5} s\right)\left(\bar{d} i \gamma_{5} u\right)$ \\
$\bar{M}_{s} \pi$ & $0^{+}$ & $\left(\bar{Q} i \gamma_{\mu} u\right)\left(\bar{d} i \gamma_{5} s\right)$ \\
$\bar{M}^{*} K$ & $1^{+}$ & $\left(\bar{Q} i \gamma_{\mu} s\right)\left(\bar{d} i \gamma_{5} u\right)$ \\
$\bar{M}_{s}^{*} \pi$ & $1^{+}$ & $\left(s^{T} C \gamma_{5} u\right)\left(\bar{Q} \gamma_{\mu} \gamma_{5} C \bar{d}^{T}\right)+k\left(s^{T} C u\right)\left(\bar{Q} \gamma_{\mu} C \bar{d}^{T}\right)$ \\
Four-quark & & $\left(s^{T} C \gamma_{5} u\right)\left(\bar{Q} \gamma_{\mu} C \bar{d}^{T}\right)+k\left(s^{T} C u\right)\left(\bar{Q} \gamma_{\mu} \gamma_{5} C \bar{d}^{T}\right)$ \\
& $1^{-}$ & \\
\hline \hline
\end{tabular}


- Spectral function within MDA

We shall use the Minimal Duality Ansatz (MDA) for parametrizing the spectral function:

$\frac{1}{\pi} \operatorname{Im} \Pi_{m o l}(t) \simeq f_{H}^{2} M_{H}^{8} \delta\left(t-M_{H}^{2}\right)+$ “QCD continuum" $\theta\left(t-t_{c}\right)$,

where $f_{H}$ is the decay constant defined as:

$$
\left\langle 0\left|O_{m o l}\right| H\right\rangle=f_{H} M_{H}^{4},\left\langle 0\left|O_{m o l}^{\mu}\right| H\right\rangle=f_{H} M_{H}^{5} \epsilon_{\mu},
$$

respectively for spin 0 and 1 states with $\epsilon_{\mu}$ the (axial-) vector polarization. Noting that in the previous definition in Tables 1 3 , the bilinear (pseudo)scalar current acquires an anomalous dimension due to its normalization, thus the decay constants run to order $\alpha_{s}^{2}$ as ${ }^{4}$.

$$
\begin{aligned}
f_{m o l}^{(s, p)}(\mu) & =\hat{f}_{m o l}^{(s, p)}\left(-\beta_{1} a_{s}\right)^{4 / \beta_{1}} / r_{m}^{2}, \\
f_{m o l}^{(1)}(\mu) & =\hat{f}_{m o l}^{(1)}\left(-\beta_{1} a_{s}\right)^{2 / \beta_{1}} / r_{m},
\end{aligned}
$$

where we have introduced the renormalization group invariant coupling $\hat{f}_{m o l} ;-\beta_{1}=(1 / 2)\left(11-2 n_{f} / 3\right)$ is the first coefficient of the QCD $\beta$-function for $n_{f}$ flavours and $a_{s} \equiv\left(\alpha_{s} / \pi\right)$. The QCD corrections numerically read;

$$
\begin{gathered}
r_{m}\left(n_{f}=4\right)=1+1.014 a_{s}+1.389 a_{s}^{2}, \\
r_{m}\left(n_{f}=5\right)=1+1.176 a_{s}+1.501 a_{s}^{2} .
\end{gathered}
$$

The higher order states contributions are smeared by the "QCD continuum" coming from the discontinuity of the QCD diagrams and starting from a constant threshold $\sqrt{t_{c}}$.

\section{- NLO and N2LO PT corrections using factorization}

Assuming a factorization of the four-quark interpolating current as a natural consequence of the molecule definition of the state, we can write the corresponding spectral function as a convolution of the ones associated to quark bilinear current. In this way, we obtain [19] for the $\bar{D} D^{*}$ and $\bar{D}_{0}^{*} D^{*}$-like spin 1 states:

$$
\begin{aligned}
\frac{1}{\pi} \operatorname{Im} \Pi_{m o l}^{(1)}(t)= & \theta\left(t-4 M_{Q}^{2}\right)\left(\frac{1}{4 \pi}\right)^{2} t^{2} \int_{M_{Q}^{2}}^{\left(\sqrt{t}-M_{Q}\right)^{2}} d t_{1} \int_{M_{Q}^{2}}^{\left(\sqrt{t}-\sqrt{t_{1}}\right)^{2}} d t_{2} \\
& \times \lambda^{3 / 2} \frac{1}{\pi} \operatorname{Im} \Pi^{(1)}\left(t_{1}\right) \frac{1}{\pi} \operatorname{Im} \psi^{(s, p)}\left(t_{2}\right) .
\end{aligned}
$$

For the $\bar{D} D$ spin 0 state, one has:

$$
\begin{aligned}
& \frac{1}{\pi} \operatorname{Im} \psi_{m o l}^{(s)}(t)=\theta\left(t-4 M_{Q}^{2}\right)\left(\frac{1}{4 \pi}\right)^{2} t^{2} \int_{M_{Q}^{2}}^{\left(\sqrt{t}-M_{Q}\right)^{2}} d t_{1} \int_{M_{Q}^{2}}^{\left(\sqrt{t}-\sqrt{t_{1}}\right)^{2}} d t_{2} \\
& \times \lambda^{1 / 2}\left(\frac{t_{1}}{t}+\frac{t_{2}}{t}-1\right)^{2} \frac{1}{\pi} \operatorname{Im} \psi^{(p)}\left(t_{1}\right) \frac{1}{\pi} \operatorname{Im} \psi^{(p)}\left(t_{2}\right),
\end{aligned}
$$

\footnotetext{
${ }^{4}$ The coupling of the (pseudo)scalar molecule built from two (axial)-vector currents has no anomalous dimension and does not run.
}

and for the $\bar{D}^{*} D^{*}$ spin 0 state:

$$
\begin{aligned}
\frac{1}{\pi} \operatorname{Im} \psi_{m o l}^{(s)}(t)= & \theta\left(t-4 M_{Q}^{2}\right)\left(\frac{1}{4 \pi}\right)^{2} t^{2} \int_{M_{Q}^{2}}^{\left(\sqrt{t}-M_{Q}\right)^{2}} d t_{M_{Q}^{2}}^{\left(\sqrt{t}-\sqrt{t_{1}}\right)^{2}} d t_{2} \\
& \times \lambda^{1 / 2} \times\left[\left(\frac{t_{1}}{t}+\frac{t_{2}}{t}-1\right)^{2}+\frac{8 t_{1} t_{2}}{t^{2}}\right] \\
& \times \frac{1}{\pi} \operatorname{Im} \Pi^{(1)}\left(t_{1}\right) \frac{1}{\pi} \operatorname{Im} \Pi^{(1)}\left(t_{2}\right),(10)
\end{aligned}
$$

where:

$$
\lambda=\left(1-\frac{\left(\sqrt{t_{1}}-\sqrt{t_{2}}\right)^{2}}{t}\right)\left(1-\frac{\left(\sqrt{t_{1}}+\sqrt{t_{2}}\right)^{2}}{t}\right),
$$

is the phase space factor and $M_{Q}$ is the on-shell heavy quark mass. $\operatorname{Im} \Pi^{(1)}(t)$ is the spectral function associated to the bilinear $\bar{c} \gamma_{\mu}\left(\gamma_{5}\right) q$ (axial-)vector current, while $\operatorname{Im} \psi^{(s, p)}(t)$ is associated to the $\bar{c} i\left(\gamma_{5}\right) q$ (pseudo)scalar current We shall assume that a such factorization also holds for four-quark states.

\section{- The inverse Laplace transform sum rule (LSR)}

The LSR and its ratio read:

$$
\begin{aligned}
& \mathcal{L}_{m o l}\left(\tau, t_{c}, \mu\right)=\frac{1}{\pi} \int_{4 M_{Q}^{2}}^{t_{c}} d t e^{-t \tau} \operatorname{Im}\left\{\Pi_{m o l} ; \psi_{m o l}\right\}(t, \mu), \\
& \mathcal{R}_{m o l}\left(\tau, t_{c}, \mu\right)=\frac{\int_{4 M_{Q}^{2}}^{t_{c}} d t t e^{-t \tau} \operatorname{Im}\left\{\Pi_{m o l} ; \psi_{m o l}\right\}(t, \mu)}{\int_{4 M_{Q}^{2}}^{t_{c}} d t e^{-t \tau} \operatorname{Im}\left\{\Pi_{m o l} ; \psi_{m o l}\right\}(t, \mu)} \simeq M_{R}^{2},
\end{aligned}
$$

where $\mu$ is the subtraction point which appears in the approximate QCD series when radiative corrections are included and $\tau$ is the sum rule variable replacing $q^{2}$.

\section{- Double ratios of inverse Laplace transform sum rule}

Double Ratios of Sum Rules (DRSR) [9, 10, 20, 29] are also useful for extracting the SU3 breaking effects on couplings and mass ratios. They read:

$$
f_{m o l}^{s d} \equiv \frac{\mathcal{L}_{m o l}^{s}\left(\tau, t_{c}, \mu\right)}{\mathcal{L}_{m o l}^{d}\left(\tau, t_{c}, \mu\right)}, \quad r_{m o l}^{s d} \equiv \frac{\mathcal{R}_{m o l}^{s}\left(\tau, t_{c}, \mu\right)}{\mathcal{R}_{m o l}^{d}\left(\tau, t_{c}, \mu\right)},
$$

where the upper indices $s, d$ indicates the $s$ and $d$ quark channels. These DRSR can be used when each sum rule optimizes at the same values of the parameters $\left(\tau, t_{c}, \mu\right)$.

\footnotetext{
${ }^{5}$ In the chiral limit $m_{q}=0$, the PT expressions of the vector (resp. scalar) and axial-vector (resp. pseudoscalar) spectral functions are the same.
} 


\section{- Stability criteria and some phenomenological tests}

The variables $\tau, \mu$ and $t_{c}$ are, in principle, free external parameters. We shall use stability criteria (if any) with respect to these free 3 parameters, for extracting the optimal results. In the standard MDA given in Eq.4 for parametrizing the spectral function, the "QCD continuum" threshold $\sqrt{t_{c}}$ is constant and is independent on the subtraction point $\mu$. One should notice that this standard MDA with constant $\sqrt{t_{c}}$ describes quite well the properties of the lowest ground state as explicitly demonstrate in [30] and in various examples [9, 10] after confronting the integrated spectral function within this simple parametrization with the full data measurements. It has been also successfully tested in the large $N_{c}$ limit of QCD in [31]. Though it is difficult to estimate with a good precision the systematic error related to this simple model for reproducing accurately the data, we expect that the same feature is reproduced for the case of the XYZ discussed here where complete data are still lacking.

\section{QCD input parameters}

The QCD parameters which shall appear in the following analysis will be the charm and bottom quark masses $m_{c, b}$, the strange quark mass $m_{s}$ (we shall neglect the light quark masses $m_{u, d}$ ), the light quark condensate $\langle\bar{q} q\rangle(q \equiv u, d)$, the gluon condensates $\left\langle\alpha_{s} G^{2}\right\rangle \equiv\left\langle\alpha_{s} G_{\mu \nu}^{a} G_{a}^{\mu \nu}\right\rangle$ and $\left\langle g^{3} G^{3}\right\rangle \equiv\left\langle g^{3} f_{a b c} G_{\mu \nu}^{a} G_{\nu \rho}^{b} G_{\rho \mu}^{c}\right\rangle$, the mixed condensate $\langle\bar{q} G q\rangle \equiv\left\langle\bar{q} g \sigma^{\mu \nu}\left(\lambda_{a} / 2\right) G_{\mu \nu}^{a} q\right\rangle=$ $M_{0}^{2}\langle\bar{q} q\rangle$ and the four-quark condensate $\rho \alpha_{s}\langle\bar{q} q\rangle^{2}$, where $\rho \simeq 3-4$ indicates the deviation from the four-quark vacuum saturation. Their values are given in Table 4 and more recently in [32]. The original errors on $\kappa \equiv$ $\langle\bar{s} s\rangle /\langle\bar{d} d\rangle$ have been enlarged to take into account the lattice result [33] which needs to be checked by some other groups. We shall work with the running light quark condensates, which read to leading order in $\alpha_{s}$ :

$$
\begin{aligned}
\langle\bar{q} q\rangle(\tau) & =-\hat{\mu}_{q}^{3}\left(-\beta_{1} a_{s}\right)^{2 / \beta_{1}} \\
\langle\bar{q} G q\rangle(\tau) & =-M_{0}^{2} \hat{\mu}_{q}^{3}\left(-\beta_{1} a_{s}\right)^{1 / 3 \beta_{1}}
\end{aligned}
$$

and the running quark mass to NLO (for the number of flavours $n_{f}=3$ )

$$
\bar{m}_{s}(\tau)=\hat{m}_{s}\left(-\beta_{1} a_{s}\right)^{-2 / \beta_{1}}\left(1+0.8951 a_{s}\right)
$$

where $\hat{\mu}_{q}$ and $\hat{m}_{s}$ is the spontaneous RGI light quark condensate [34] and strange quark mass.
Table 4: QCD input parameters:the original errors for $\left\langle\alpha_{s} G^{2}\right\rangle,\left\langle g^{3} G^{3}\right\rangle$

\begin{tabular}{|c|c|c|}
\hline Parameters & Values & Ref. \\
\hline$\overline{\alpha_{s}\left(M_{\tau}\right)}$ & $0.325(8)$ & $|35+| 38 \mid$ \\
\hline$\hat{m}_{s}$ & $(0.114 \pm 0.006) \mathrm{GeV}$ & \begin{tabular}{|l|l|l|l|l|l|}
9 & 20 & 22 & 35 & 39 & 41 \\
\end{tabular} \\
\hline $\bar{m}_{c}\left(m_{c}\right)$ & 1261(12) MeV & average [42, 48 \\
\hline $\bar{m}_{b}\left(m_{b}\right)$ & 4177(11) MeV & average 42,46 \\
\hline$\hat{\mu}_{q}$ & $(253 \pm 6) \mathrm{MeV}$ & \begin{tabular}{|l|l|l|l|l|}
9 & 20 & 22 & 39 & 41 \\
\end{tabular} \\
\hline$\kappa \equiv\langle\bar{s} s\rangle /\langle\bar{d} d\rangle$ & $\left(0.74_{-012}^{+0.34}\right)$ & $\begin{array}{lll}9 & 27 & 28 \\
\end{array}$ \\
\hline$M_{0}^{2}$ & $(0.8 \pm 0.2) \mathrm{GeV}^{2}$ & [21 $49-53$ \\
\hline$\left\langle\alpha_{s} G^{2}\right\rangle$ & $(7 \pm 3) \times 10^{-2} \mathrm{GeV}^{4}$ & 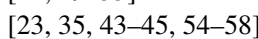 \\
\hline$\left\langle g^{3} G^{3}\right\rangle$ & $(8.2 \pm 2.0) \mathrm{GeV}^{2} \times\left\langle\alpha_{s} G^{2}\right\rangle$ & 43, 45 \\
\hline$\rho \alpha_{s}\langle\bar{q} q\rangle^{2}$ & $(5.8 \pm 1.8) \times 10^{-4} \mathrm{GeV}^{6}$ & \begin{tabular}{|l|l|l|l|}
35 & 49 & 51 & 54 \\
\end{tabular} \\
\hline
\end{tabular}
and $\rho\langle\bar{q} q\rangle^{2}$ have been multiplied by about a factor 3 for a conservative estimate of the errors (see also the text).

\section{QCD expressions of the spectral functions}

In our works [1-3], we provide new compact integrated expressions of QCD spectral functions at LO of PT QCD and including non-perturbative (NP) condensates having dimensions $d \leq 6-8$. NLO and N2LO corrections are introduced using the convolution integrals in Eq.8. The expressions of the QCD spectral functions of heavy-light bilinear currents are known to or$\operatorname{der} \alpha_{s}$ (NLO) from [59] and to order $\alpha_{s}^{2}$ (N2LO) in the chiral limit $m_{q}=0$ from [60, 61] which are available as a Mathematica program named Rvs. We shall use the SU3 breaking PT corrections at NLO [62] from the two-point function formed by bilinear currents. N3LO corrections are estimated from a geometric growth of the QCD PT series [63] as a source of PT errors, which we expect to give a good approximation of the uncalculated higher order terms dual to the $1 / q^{2}$ contribution of a tachyonic gluon mass [64, 65].

In our analysis, we replace the on-shell (pole) mass appearing in the spectral functions with the running mass using the relation, to order $\alpha_{s}^{2}$ [9-11]:

$$
\begin{aligned}
M_{Q}= & \bar{m}_{Q}(\mu)\left[1+\frac{4}{3} a_{s}+\left(16.2163-1.0414 n_{l}\right) a_{s}^{2}\right. \\
& +\log \left(\frac{\mu}{M_{Q}}\right)^{2}\left(a_{s}+\left(8.8472-0.3611 n_{l}\right) a_{s}^{2}\right) \\
& \left.+\log ^{2}\left(\frac{\mu}{M_{Q}}\right)^{2}\left(1.7917-0.0833 n_{l}\right) a_{s}^{2} \ldots\right],(17)
\end{aligned}
$$

for $n_{l}$ light flavours where $\mu$ is the arbitrary subtraction point.

\section{Tests of the Factorization Assumption}

- Factorization test for PT $\oplus$ NP contributions at LO

From our previous work [2, 16], we have noticed that assuming a factorization of the PT at LO and including 


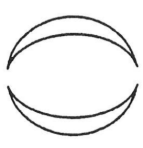

(a)

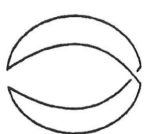

(b)
Figure 1: (a) Factorized contribution to the four-quark correlator at lowest order of PT; (b) Non-factorized contribution at lowest order of PT (the figure comes from [19]).

NP contributions induces an effect about $2.2 \%$ for the decay constant and $0.5 \%$ for the mass, which is quite tiny. However, we shall work in the following with the full non-factorized PT $\oplus$ NP of the LO expressions.

- Test at NLO of PT from the $\bar{B}^{0} B^{0}$ four-quark correlator

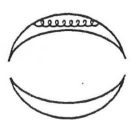

(a)

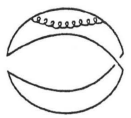

(d)

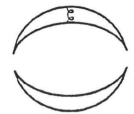

(b)

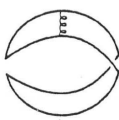

(e)

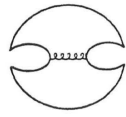

(c)

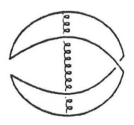

(f)
Figure 2: (a,b) Factorized contribution to the four-quark correlator at NLO of PT; (c to f) Non-factorized contribution at NLO of PT (the figure comes from [19]).

For extracting the PT $\alpha_{s}^{n}$ corrections to the correlator and due to the technical complexity of the calculations, we shall assume that these radiative corrections are dominated by the ones from factorized diagrams while we neglect the ones from non-factorized diagrams. This fact has been proven explicitly by $[66,67$ in the case of $\bar{B}^{0} B^{0}$ systems (very similar correlator as the ones discussed in the following) where the nonfactorized $\alpha_{s}$ corrections do not exceed $10 \%$ of the total $\alpha_{s}$ contributions

\section{- Conclusions of the factorization tests}

We expect from the previous LO examples that the masses of the molecules are known with a good accuracy while, for the coupling, we shall have in mind the systematics induced by the radiative corrections estimated by keeping only the factorized diagrams where their contributions will be extracted from the convolution integrals given in Eq. 5. Here, the suppression of the NLO corrections will be more pronounced for the extraction of the meson masses from the ratio of sum rules compared to the case of the $\bar{B}^{0} B^{0}$ systems.

\section{Molecules and four-quark states}

We shall study the charm channels and their beauty analogue in chiral limit (resp. SU3 breaking). As the analysis will be performed using the same techniques, we shall illustrate it in the case of $\bar{D} D\left(\right.$ resp. $\left.\bar{D}_{s} D_{s}\right)$. The results are given in Tables 5 to 7 (resp. Tables 8 to 10) for the chiral limit (resp. SU3 breaking) case.

\section{- $\bar{D} D$ state in chiral limit}

We study the behavior of the coupling ${ }^{6}$ and mass in term of LSR variable $\tau$ for different values of $t_{c}$ at N2LO as shown in Fig. 3 We consider as final and conserva-

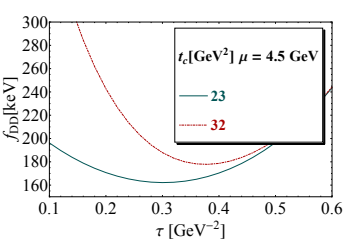

a)

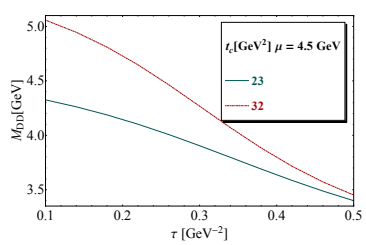

b)
Figure 3: a) The coupling $f_{D D}$ at N2LO as function of $\tau$ for different values of $t_{c}$, for $\mu=4.5 \mathrm{GeV}$ and for the QCD parameters in Table 4 b) The same as a) but for the mass $M_{D D}$.

tive results the one corresponding to the beginning of the $\tau$ stability for $t_{c}=23 \mathrm{GeV}^{2}$ and $\tau \simeq 0.25 \mathrm{GeV}^{-2}$ until the one where $t_{c}$ stability is reached for $t_{c} \simeq 32 \mathrm{GeV}^{2}$ and $\tau \simeq 0.35 \mathrm{GeV}^{-2}$.

\section{- Convergence of the PT series}

Using the previous value of $t_{c} \simeq 32 \mathrm{GeV}^{2}$, we study in Fig. 4 the convergence of the PT series for a given value of $\mu=4.5 \mathrm{GeV}$. We observe that from NLO to N2LO, the mass decreases by $\sim 0.1 \%$. This result indicates a good convergence of the PT series.

\section{$-\mu$-stability}

We improve our previous results by using different values of $\mu$ (Fig. 5). Using the fact that the final results must be independent of the arbitrary parameter $\mu$, we consider as optimal results the one at the inflexion point for $\mu \simeq(4.0-4.5) \mathrm{GeV}$.

\footnotetext{
${ }^{6}$ Here and in the following: decay constant is the same as coupling
} 
Table 5: $\bar{D} D$-like molecules masses, invariant and running couplings from LSR within stability criteria at LO to N2LO of PT. The invariant coupling $\hat{f}_{X}$ is defined in Eq. 6

\begin{tabular}{|c|c|c|c|c|c|c|c|c|c|c|c|}
\hline \multirow[t]{2}{*}{ Nature } & \multicolumn{3}{|c|}{$\hat{f}_{X}[\mathrm{keV}]$} & \multicolumn{3}{|c|}{$f_{X}(4.5)[\mathrm{keV}]$} & \multicolumn{3}{|c|}{ Mass [MeV] } & \multirow[t]{2}{*}{ Threshold } & \multirow[t]{2}{*}{ Exp. } \\
\hline & LO & NLO & N2LO & LO & NLO & N2LO & LO & NLO & N2LO & & \\
\hline$\overline{\mathbf{J}}^{\mathrm{PC}}=\mathbf{0}^{++}$ & & & & & & & & & & & - \\
\hline $\bar{D} D$ & 56 & 60 & $62(6)$ & 155 & 164 & $170(15)$ & 3901 & 3901 & $3898(36)$ & 3739 & \\
\hline $\bar{D}^{*} D^{*}$ & - & - & - & 269 & 288 & $302(47)$ & 3901 & 3903 & $3903(179)$ & 4020 & \\
\hline$D_{0}^{*} D_{0}^{*}$ & - & - & - & - & 97 & $114(18)$ & - & 4003 & $3954(223)$ & 4636 & \\
\hline $\bar{D}_{1} D_{1}$ & - & - & - & - & 236 & 274(37) & - & 3838 & $3784(56)$ & - & \\
\hline $\mathbf{J}^{\mathbf{P C}}=\mathbf{1}^{+ \pm}$ & & & & & & & & & & & $X_{c}, Z_{c}$ \\
\hline $\bar{D}^{*} D$ & 87 & 93 & $97(10)$ & 146 & 154 & $161(17)$ & 3901 & 3901 & $3903(62)$ & 3880 & \\
\hline $\bar{D}_{0}^{*} D_{1}$ & - & - & - & - & 96 & $112(17)$ & - & 3849 & $3854(182)$ & 4739 & \\
\hline $\mathbf{J}^{\mathbf{P C}}=\mathbf{0}^{- \pm}$ & & & & & & & & & & & - \\
\hline $\bar{D}_{0}^{*} D$ & 68 & 88 & 94(7) & 190 & 240 & 257(19) & 5956 & 5800 & $5690(140)$ & 4188 & \\
\hline $\bar{D}^{*} D_{1}$ & - & - & - & 382 & 490 & $564(38)$ & 6039 & 5898 & $5797(141)$ & 4432 & \\
\hline $\mathbf{J}^{\mathbf{P C}}=\mathbf{1}^{--}$ & & & & & & & & & & & $Y_{c}$ \\
\hline $\bar{D}_{0}^{*} D^{*}$ & 112 & 143 & $157(10)$ & 186 & 238 & 261(17) & 6020 & 5861 & $5748(101)$ & 4328 & \\
\hline $\begin{array}{l}\bar{D} D_{1} \\
\mathbf{J}^{\mathbf{P C}}=\mathbf{1}^{-+}\end{array}$ & 98 & 126 & $139(13)$ & 164 & 209 & $231(21)$ & 5769 & 5639 & $5544(162)$ & 4291 & $Y_{c}$ \\
\hline $\bar{D}_{0}^{*} D^{*}$ & 105 & 135 & $150(13)$ & 174 & 224 & $249(22)$ & 6047 & 5920 & $5828(132)$ & 4328 & \\
\hline $\bar{D} D_{1}$ & 97 & 128 & $145(15)$ & 162 & 213 & 241(25) & 5973 & 5840 & $5748(179))$ & - & \\
\hline
\end{tabular}

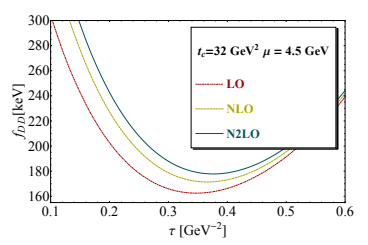

a)

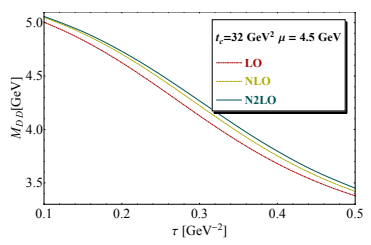

b)

Figure 4: a) $\tau$-behavior of $f_{\bar{D} D}$ for $t_{c}=32 \mathrm{GeV}^{2}$ and $\mu=4.5 \mathrm{GeV}$ and for different truncation of the PT series; b) the same as a) but for $M_{\bar{D} D}$.

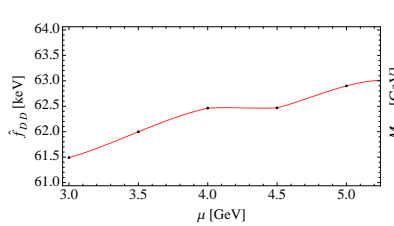

a)

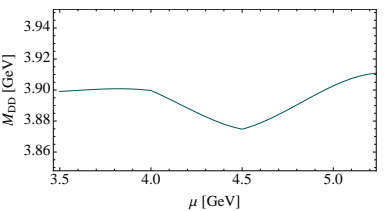

b)

Figure 5: a) $\mu$-behavior of $\hat{f}_{\bar{D} D}$ at N2LO; b) $\mu$-behavior of $M_{\bar{D} D}$ at N2LO.

\section{- $\bar{D} D$-SU3 breaking $\left(\bar{D}_{s} D_{s}\right)$}

The analysis of the $\mu$ subtraction point is very similar to the previous one and will not be repeated here. Using the optimal choice $\mu=4.5 \mathrm{GeV}$ obtained previously, we study the behaviour of the coupling and mass in term of LSR variable $\tau$ for different values of $t_{c}$ at NLO (see Fig.6.

\section{- SU3 ratios of masses and couplings}

We study the behaviour of the SU3 ratios of couplings $\left(f_{D D}^{s d}\right)$ and masses $\left(r_{D D}^{s d}\right)$ in terms of LSR vari-

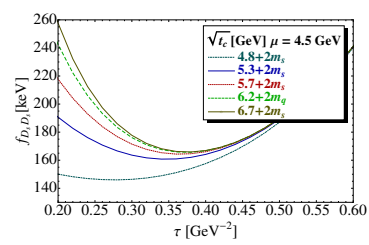

a)

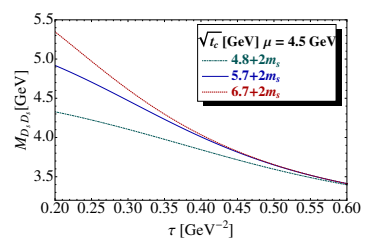

b)
Figure 6: a) The coupling $f_{D_{s} D_{s}}$ at NLO as function of $\tau$ for different values of $t_{c}$, for $\mu=4.5 \mathrm{GeV}$ and for the QCD parameters in Table 4 b) The same as a) but for the mass $M_{D_{s} D_{s}}$.

able $\tau$ for different values of $t_{c}$ at NLO as shown in Fig.7. We consider as an optimal estimate the mean

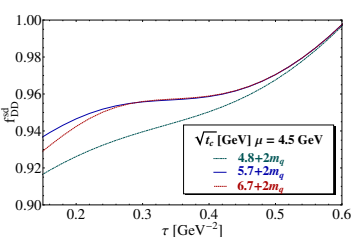

a)

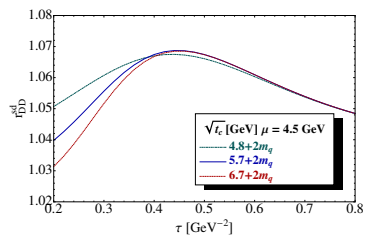

b)
Figure 7: a) The SU3 ratio of couplings $f_{D D}^{s d}$ at NLO as function of $\tau$ for different values of $t_{c}$, for $\mu=4.5 \mathrm{GeV}$ and for the QCD parameters in Table 4 b) The same as a) but for the ratio of mass $r_{D D}^{s d}$.

value of the coupling, mass and their SU3 ratios obtained at the minimum or inflexion point for the common range of $t_{c}$-values $\left(\sqrt{t_{c}} \simeq 4.8+2 \bar{m}_{s} \mathrm{GeV}\right.$ for $\left.\tau \simeq(0.28 \pm 0.02) \mathrm{GeV}^{-2}\right)$ corresponding to the starting of the $\tau$-stability and the one where (almost) $t_{c^{-}}$ 
Table 6: $\bar{B} B$-like molecules masses, invariant and running couplings from LSR within stability criteria from LO to N2LO of PT. The invariant coupling $\hat{f}_{X}$ is defined in Eq. 6

\begin{tabular}{|c|c|c|c|c|c|c|c|c|c|c|c|}
\hline \multirow[t]{2}{*}{ Nature } & \multicolumn{3}{|c|}{$\hat{f}_{X}[\mathrm{keV}]$} & \multicolumn{3}{|c|}{$f_{X}(5.5)[\mathrm{keV}]$} & \multicolumn{3}{|c|}{ Mass $[\mathrm{MeV}]$} & \multirow[t]{2}{*}{ Threshold } & \multirow[t]{2}{*}{ Exp. } \\
\hline & LO & NLO & N2LO & LO & NLO & $\mathrm{N} 2 \mathrm{LO}$ & LO & NLO & N2LO & & \\
\hline$\overline{\mathbf{J}}^{\mathrm{PC}}=\mathbf{0}^{++}$ & & & & & & & & & & & - \\
\hline $\bar{B} B$ & 4.0 & 4.4 & $5(1)$ & 14.4 & 15.6 & $17(4)$ & 10605 & 10598 & $10595(58)$ & 10559 & \\
\hline $\bar{B}^{*} B^{*}$ & - & - & - & 27 & 30 & $32(5)$ & 10626 & 10646 & $10647(184)$ & 10650 & \\
\hline$B_{0}^{*} B_{0}^{*}$ & 2.1 & 3.2 & $4(1)$ & 7.7 & 11.3 & $14(4)$ & 10653 & 10649 & $10648(113)$ & - & \\
\hline $\bar{B}_{1} B_{1}$ & - & - & - & - & 20 & $28.6(4)$ & - & 10514 & $10514(149)$ & - & \\
\hline $\mathbf{J}^{\mathbf{P C}}=\mathbf{1}^{+ \pm}$ & & & & & & & & & & & $X_{b}, Z_{b}$ \\
\hline $\bar{B}^{*} B$ & 7 & 8 & $9(3)$ & 14 & 16 & $17(5)$ & 10680 & 10673 & $10646(150)$ & 10605 & \\
\hline $\bar{B}_{0}^{*} B_{1}$ & 4 & 6 & $7(1)$ & 8 & 11 & $14(2)$ & 10670 & 10679 & $10692(132)$ & - & \\
\hline $\mathbf{J}^{\mathbf{P C}}=\mathbf{0}^{- \pm}$ & & & & & & & & & & & - \\
\hline $\bar{B}_{0}^{*} B$ & 11 & 16 & 20(3) & 39 & 55 & $67(10)$ & 12930 & 12737 & $12562(260)$ & - & \\
\hline $\bar{B}^{*} B_{1}$ & - & - & - & 71 & 105 & 136(19) & 12967 & 12794 & $12627(225)$ & 11046 & \\
\hline $\mathbf{J}^{\mathbf{P C}}=\mathbf{1}^{--}$ & & & & & & & & & & & $Y_{b}$ \\
\hline $\bar{B}_{0}^{*} B^{*}$ & 21 & 29 & $35(6)$ & 39 & 54 & $66(11)$ & 12936 & 12756 & $12592(266)$ & - & \\
\hline $\begin{array}{l}\bar{B} B_{1} \\
\mathbf{J}^{\mathbf{P C}}=\mathbf{1}^{-+}\end{array}$ & 21 & 29 & $35(7)$ & 39 & 54 & $65(12)$ & 12913 & 12734 & $12573(257)$ & 11000 & \\
\hline $\bar{B}_{0}^{*} B^{*}$ & 20 & 29 & $34(4)$ & 38 & 54 & $64(8)$ & 12942 & 12774 & $12617(220)$ & - & $Y_{b}$ \\
\hline $\bar{B} B_{1}$ & 20 & 29 & $35(5)$ & 37 & 53 & $65(9)$ & 12974 & 12790 & $12630(236)$ & 11000 & \\
\hline
\end{tabular}

Table 7: Four-quark masses, invariant and running couplings from LSR within stability criteria from LO to N2LO of PT. The decay constant is evaluated at 4.5 (resp. 5.5) GeV for the $c$ (resp. $b$ ) channel. The invariant coupling $\hat{f}_{X}$ is defined in Eq. 6

\begin{tabular}{|c|c|c|c|c|c|c|c|c|c|c|}
\hline \multirow[t]{2}{*}{ Nature } & \multicolumn{3}{|c|}{$\hat{f}_{X}[\mathrm{keV}]$} & \multicolumn{3}{|c|}{$f_{X}[\mathrm{keV}]$} & \multicolumn{3}{|c|}{ Mass $[\mathrm{MeV}]$} & \multirow[t]{2}{*}{ Exp. } \\
\hline & LO & NLO & N2LO & LO & NLO & N2LO & LO & NLO & N2LO & \\
\hline \multicolumn{11}{|l|}{$\overline{c \text {-quark }}$} \\
\hline$S_{c}\left(0^{+}\right)$ & 62 & 67 & $70(7)$ & 173 & 184 & $191(20)$ & 3902 & 3901 & $3898(54)$ & - \\
\hline$A_{c}\left(1^{+}\right)$ & 100 & 106 & $112(18)$ & 166 & 176 & $184(30)$ & 3903 & 3890 & $3888(130)$ & $X_{c}, Z_{c}$ \\
\hline$\pi_{c}\left(0^{-}\right)$ & 84 & 106 & $113(5)$ & 233 & 292 & $310(13)$ & 6048 & 5872 & $5750(127)$ & - \\
\hline$V_{c}\left(1^{-}\right)$ & 123 & 162 & $178(11)$ & 205 & 268 & 296(19) & 6062 & 5904 & $5793(122)$ & $Y_{c}$ \\
\hline \multicolumn{11}{|l|}{$b$-quark } \\
\hline$S_{b}\left(0^{+}\right)$ & 4.6 & 5.0 & $5.3(1.1)$ & 16 & 17 & $19(4)$ & 10652 & 10653 & 10654(109) & - \\
\hline$A_{b}\left(1^{+}\right)$ & 8.7 & 9.5 & $10(2)$ & 16 & 18 & $19(3)$ & 10730 & 10701 & $10680(172)$ & $Z_{b}$ \\
\hline$\pi_{b}\left(0^{-}\right)$ & 18 & 23 & $27(3)$ & 62 & 83 & $94(11)$ & 13186 & 12920 & $12695(254)$ & - \\
\hline$V_{b}\left(1^{-}\right)$ & 24 & 33 & $40(5)$ & 45 & 62 & $75(9)$ & 12951 & 12770 & $12610(242)$ & $Y_{b}$ \\
\hline
\end{tabular}

stability $\left(\sqrt{t_{c}} \simeq 6.7+2 \bar{m}_{s} \mathrm{GeV}\right)$ is reached for $\tau \simeq$ $(0.38 \pm 0.02) \mathrm{GeV}^{-2}$.

\section{Confrontation with some LO results and data}

\section{- Comparison with some previous LO QSSR results}

The comparison is only informative as it is known that the LO results suffer from the ill-defined definition of the heavy quark mass used in the analysis at this order. Most of the authors (see e.g [68,-72]) use the running mass value which is not justified when one implicitly uses the QCD expression obtained within the on-shell scheme. The difference between some results is also due to the way for extracting the optimal information from the analysis. Here we use well-defined stability criteria verified from the example of the harmonic oscillator in quantum mechanics and from different well-known hadronic channels [9, 10].

\section{- Confrontation with experiments}

We conclude from the previous analysis that:

- For the chiral limit case, one can notice that the masses of the $J^{P}=0^{+}, 1^{+}$states are most of them below the corresponding $D D, B B$-like thresholds and are compatible with some of the observed XZ masses, suggesting that these states can be interpreted as almost pure molecules or/and four-quark states.

- The masses predictions of $\bar{D} D$, and $\bar{D}^{*} D^{*}$ molecules are compatible with the $0^{++} Z_{c}(3900)$ experimental candidate .

- The interpretation of the $0^{++}$candidates as pure fourquark ground states is not favoured by our result.

- The $0^{++} \mathrm{X}(4700)$ experimental candidate might be identified with a $\bar{D}_{s 0}^{*} D_{s 0}^{*}$ molecule ground state.

- The $1^{++} \mathrm{X}(4147)$ and X(4273) are compatible within the error with the one of the $\bar{D}_{s}^{*} D_{s}$ molecule state and with the one of the axial-vector $A_{c}$ four-quark state. 
Table 8: $\bar{D} D$-like molecules couplings, masses and their corresponding SU3 ratios from LSR within stability criteria at NLO to N2LO of PT.

\begin{tabular}{|c|c|c|c|c|c|c|c|c|}
\hline \multirow[t]{2}{*}{ Channels } & \multicolumn{2}{|c|}{$f_{M}^{s d} \equiv f_{M_{S}} / f_{M}$} & \multicolumn{2}{|c|}{$f_{M_{S}}(4.5)[\mathrm{keV}]$} & \multicolumn{2}{|c|}{$r_{M}^{s d} \equiv M_{M_{S}} / M_{M}$} & \multicolumn{2}{|c|}{$M_{M_{S}}[\mathrm{MeV}]$} \\
\hline & NLO & N2LO & NLO & N2LO & NLO & N2LO & NLO & N2LO \\
\hline Scalar $\left(0^{++}\right)$ & & & & & & & & \\
\hline $\bar{D}_{s} D_{s}$ & $0.95(3)$ & $0.98(4)$ & $156(17)$ & $167(18)$ & $1.069(4)$ & $1.070(4)$ & $4169(48)$ & $4169(48)$ \\
\hline $\bar{D}_{s}^{*} D_{s}^{*}$ & $0.93(3)$ & $0.95(3)$ & $265(31)$ & $284(34)$ & $1.069(3)$ & $1.075(3)$ & $4192(200)$ & $4196(200)$ \\
\hline $\bar{D}_{s 0}^{*} D_{s 0}^{*}$ & $0.88(6)$ & $0.89(6)$ & $85(12)$ & $102(14)$ & $1.069(69)$ & $1.058(68)$ & $4277(134)$ & $4225(132)$ \\
\hline $\bar{D}_{s 1}^{s 0} D_{s 1}^{s 0}$ & $0.906(33)$ & $0.930(34)$ & $209(28)$ & $229(31)$ & $1.097(7)$ & $1.090(7)$ & $4187(62)$ & $4124(61)$ \\
\hline $\operatorname{Axial}\left(1^{+ \pm}\right)$ & & & & & & & & \\
\hline $\bar{D}_{s}^{*} D_{s}$ & $0.93(3)$ & $0.97(3)$ & $143(16)$ & $156(17)$ & $1.070(4)$ & $1.073(4)$ & $4174(67)$ & $4188(67)$ \\
\hline $\begin{array}{l}\bar{D}_{s 0}^{*} D_{s 1} \\
\text { Pseudo }\left(0^{- \pm}\right)\end{array}$ & $0.90(1)$ & $0.82(1)$ & $87(14)$ & $110(18)$ & $1.119(24)$ & $1.100(24)$ & $4269(205)$ & $4275(206)$ \\
\hline $\bar{D}_{s 0}^{*} D_{s}$ & $0.94(5)$ & $0.90(4)$ & $225(24)$ & $232(25)$ & $0.970(50)$ & $0.946(40)$ & $5604(223)$ & $5385(214)$ \\
\hline $\begin{array}{l}\bar{D}_{s}^{*} D_{s 1} \\
\operatorname{Vector}\left(1^{--}\right)\end{array}$ & $0.93(4)$ & $0.90(4)$ & $455(34)$ & $508(38)$ & $0.970(50)$ & $0.972(34)$ & $5724(195)$ & $5632(192)$ \\
\hline $\bar{D}_{s 0}^{*} D_{s}^{*}$ & $0.87(4)$ & $0.86(4)$ & $208(11)$ & $216(11)$ & $0.980(33)$ & $0.956(32)$ & $5708(184)$ & $5571(180)$ \\
\hline $\begin{array}{l}\bar{D}_{s} D_{s 1} \\
\operatorname{Vector}\left(1^{-+}\right)\end{array}$ & $0.97(3)$ & $0.93(3)$ & 202(12) & $213(13)$ & $0.970(33)$ & $0.951(31)$ & $5459(122)$ & $5272(120)$ \\
\hline $\bar{D}_{s 0}^{*} D_{s}^{*}$ & $0.98(5)$ & $0.92(5)$ & $219(17)$ & 231(18) & $0.963(32)$ & $0.948(32)$ & $5699(184)$ & $5528(179)$ \\
\hline $\bar{D}_{s} D_{s 1}$ & $0.92(3)$ & $0.88(3)$ & $195(13)$ & $212(14)$ & $0.959(34)$ & $0.955(34)$ & $5599(155)$ & $5487(152)$ \\
\hline
\end{tabular}

Table 9: $\bar{B} B$-like molecules couplings, masses and their corresponding SU3 ratios from LSR within stability criteria at NLO to N2LO of PT. The * indicates that the value does not come from a direct determination.

\begin{tabular}{|c|c|c|c|c|c|c|c|c|}
\hline \multirow[t]{2}{*}{ Channels } & \multicolumn{2}{|c|}{$f_{M}^{s d} \equiv f_{M_{S}} / f_{M}$} & \multicolumn{2}{|c|}{$f_{M_{S}}(5.5)[\mathrm{keV}]$} & \multicolumn{2}{|c|}{$r_{M}^{s d} \equiv M_{M_{S}} / M_{M}$} & \multicolumn{2}{|c|}{$M_{M_{S}}[\mathrm{MeV}]$} \\
\hline & NLO & N2LO & NLO & $\mathrm{N} 2 \mathrm{LO}$ & NLO & $\mathrm{N} 2 \mathrm{LO}$ & NLO & N2LO \\
\hline \multicolumn{9}{|l|}{ Scalar $\left(0^{++}\right)$} \\
\hline $\bar{B}_{s} B_{s}$ & $1.04(4)$ & $1.15(4)$ & $17(2)$ & $20(2)$ & $1.027(4)$ & $1.029(4)$ & 10884(74) & 10906(74) \\
\hline $\bar{B}_{s}^{*} B_{s}^{*}$ & $1.00(3)$ & $1.12(3)$ & $31(5)$ & $36(6)$ & $1.028(5)$ & $1.029(5)$ & 10944(134) & $10956(134)$ \\
\hline $\bar{B}_{s 0}^{*} B_{s 0}^{*}$ & $1.11(5)$ & $1.07(5)$ & $13(3)$ & $17(4)$ & $1.050(11)$ & $1.034(11)$ & $11182(227)$ & $11014(224)$ \\
\hline $\bar{B}_{s 1}^{s 0} B_{s 1}^{s 0}$ & $1.197(73)$ & $1.214(74)$ & $24(5)$ & $29(6)$ & $1.040(2)$ & $1.035(2)$ & $10935(170)$ & $10882(169)$ \\
\hline \multicolumn{9}{|l|}{$\operatorname{Axial}\left(1^{+ \pm}\right)$} \\
\hline $\bar{B}_{s}^{*} B_{s}$ & $1.01(3)$ & $1.18(4)$ & $16.7(2)$ & $20(2)$ & $1.028(4)$ & $1.030(4)$ & 10972(195) & 10972(195) \\
\hline $\begin{array}{l}\bar{B}_{s 0}^{*} B_{s 1} \\
\text { Pseudo }\left(0^{- \pm}\right)\end{array}$ & $0.80(4)$ & $0.79(4)$ & $9.1(2.2)$ & $10.7(2.6)$ & $1.052(14)$ & $1.031(14)$ & $11234(208)$ & $11021(204)$ \\
\hline $\bar{B}_{s 0}^{*} B_{s}$ & $1.06(3)$ & $1.02(3)$ & $58(3)$ & $68(4)$ & $1.00(3)^{*}$ & $1.00(3)^{*}$ & $12725(217)$ & $12509(213)$ \\
\hline $\begin{array}{l}\bar{B}_{s}^{*} B_{s 1} \\
\operatorname{Vector}\left(1^{--}\right)\end{array}$ & $0.96(4)$ & $0.95(4)$ & $100(11)$ & $118(13)$ & $1.00(3)^{*}$ & $1.00(3)^{*}$ & $12726(295)$ & $12573(292)$ \\
\hline $\bar{B}_{s 0}^{*} B_{s}^{*}$ & $0.95(3)$ & $0.90(3)$ & $51(4)$ & $59(5)$ & $1.00(3)^{*}$ & $0.99(3)^{*}$ & $12715(267)$ & $12512(263)$ \\
\hline $\begin{array}{l}\bar{B}_{s} B_{s 1} \\
\operatorname{Vector}\left(1^{-+}\right)\end{array}$ & $0.83(4)$ & $0.77(3)$ & $45(3)$ & $50(3)$ & $0.99(3)^{*}$ & $0.99(3)^{*}$ & $12615(236)$ & $12426(233)$ \\
\hline $\bar{B}_{s 0}^{*} B_{s}^{*}$ & $0.94(3)$ & $0.92(3)$ & $51(5)$ & $59(6)$ & $1.00(3)^{*}$ & $0.99(3)^{*}$ & $12734(262)$ & $12479(257)$ \\
\hline $\bar{B}_{s} B_{s 1}$ & $0.89(4)$ & $0.85(3)$ & $48(5)$ & $55(6)$ & $0.99(3) *$ & $0.98(3)^{*}$ & $12602(247)$ & $12350(242)$ \\
\hline
\end{tabular}

- Our predictions suggest the presence of $0^{++} \bar{D}_{s} D_{s}$ and $\bar{D}_{s}^{*} D_{s}^{*}$ molecule states in the range $(4121 \sim 4396) \mathrm{MeV}$ and a $\bar{D}_{s 0}^{*} D_{s 1}$ state around $4841 \mathrm{MeV}$.

- The predictions for the $J^{P}=0^{-}, 1^{-}$non-strange states are about $1.5 \mathrm{GeV}$ higher than the observed $Y_{c}$ mesons masses and $(1.7 \sim 2.6) \mathrm{GeV}$ higher than the observed $Y_{b}$ ones. Our reults do not favor their interpretation as pure molecule or/and four-quark states.

- We also present new predictions for the $0^{- \pm}, 1^{- \pm}$and for different beauty states which can be tested in future experiments.

\section{8. $\bar{B} K$ and $(\overline{Q q})(u s)$-like states and the $X(5568)$}

In [3], we have also studied the $X$ hadron formed by 3 light quarks $u d s$ and one heavy quark $Q \equiv c, b$ using the same approach as above by assuming that it is a molecule or four-quark state. We have included NLO and N2LO PT corrections and the contributions of condensates of dimension $d \leq 7$. Our results are summarized in Table 11. Contrary to previous claims in the sum rule literature, our results do not favour a $B K, B^{*} K$ or $B_{s} \pi$ molecule or four-quark $(b u)(\overline{d s})$ state having a mass around $5568 \mathrm{MeV}$ observed by D0 [73] but not confirmed by $\mathrm{LHCb}[74]$. We also predict the corresponding state in the c-quark channel where the $D_{s 0}^{*}(2317)$ seen by BABAR [75] in the $D_{s} \pi$ invariant mass, expected to be an isoscalar-scalar state with a width less than $3.8 \mathrm{MeV}$ [46] could be a good candidate for one of such states. From our analysis, one may suggest an experimental scan of the regions (2327 2444) $\mathrm{MeV}$ and $(5173 \sim 5226) \mathrm{MeV}$ for detecting these un- 
Table 10: Four-quark couplings, masses and their corresponding SU3 ratios from LSR within stability criteria at NLO and N2LO of PT. The * indicates that the value does not come from a direct determination. The decay constant is evaluated at 4.5 (resp. 5.5) GeV for the $c$ (resp. $b$ ) channel.

\begin{tabular}{|c|c|c|c|c|c|c|c|c|}
\hline \multirow[t]{2}{*}{ Channels } & \multicolumn{2}{|c|}{$f_{M}^{s d} \equiv f_{M_{S}} / f_{M}$} & \multicolumn{2}{|c|}{$f_{M_{S}}[\mathrm{keV}]$} & \multicolumn{2}{|c|}{$r_{M}^{s d} \equiv M_{M_{S}} / M_{M}$} & \multicolumn{2}{|c|}{$M_{M_{S}}[\mathrm{MeV}]$} \\
\hline & NLO & N2LO & NLO & N2LO & NLO & N2LO & NLO & N2LO \\
\hline \multicolumn{9}{|l|}{ c-quark } \\
\hline$S_{s c}\left(0^{+}\right)$ & $0.91(4)$ & $0.98(4)$ & 161(17) & 187(19) & $1.085(11)$ & $1.086(11)$ & $4233(61)$ & $4233(61)$ \\
\hline$A_{s c}\left(1^{+}\right)$ & $0.80(4)$ & $0.87(4)$ & $141(15)$ & $160(17)$ & $1.081(4)$ & $1.082(4)$ & $4205(112)$ & $4209(112)$ \\
\hline$\pi_{s c}\left(0^{-}\right)$ & $0.88(7)$ & $0.86(7)$ & $256(29)$ & $267(30)$ & $0.97(3)^{*}$ & $0.96(3)^{*}$ & $5671(181)$ & $5524(176)$ \\
\hline$V_{s c}\left(1^{-}\right)$ & $0.91(10)$ & $0.87(10)$ & $245(31)$ & $258(33)$ & $0.96(4)^{*}$ & $0.96(4)^{*}$ & $5654(239)$ & $5539(234)$ \\
\hline \multicolumn{9}{|l|}{ b-quark } \\
\hline$S_{s b}\left(0^{+}\right)$ & $0.78(3)$ & $0.83(3)$ & $22(5)$ & $26(6)$ & $1.044(4)$ & $1.048(4)$ & 11122(149) & 11133((149) \\
\hline$A_{s b}\left(1^{+}\right)$ & $0.92(3)$ & $0.98(3)$ & $22(4)$ & $26(5)$ & $1.042(6)$ & $1.046(6)$ & 11150(172) & 11172(172) \\
\hline$\pi_{s b}\left(0^{-}\right)$ & $0.80(7)$ & $0.76(4)$ & $66(12)$ & 71(13) & $0.985(2)^{*}$ & $0.975(2)^{*}$ & $12730(215)$ & 12374(209) \\
\hline$V_{s b}\left(1^{-}\right)$ & $0.97(6)$ & $0.90(6)$ & $64(8)$ & $68(9)$ & $0.996(3)^{*}$ & $0.984(30) *$ & $12716(272)$ & 12411(266) \\
\hline
\end{tabular}

Table 11: $\bar{B} K-$ and $(\overline{c u})(d s)$ )-like masses and couplings from LSR at N2LO. The running coupling $f_{X}$ is evaluated at 2-2.5 (resp. 4.5) GeV for the $c$ (resp. $b$ ) channel. The invariant coupling $\hat{f}_{X}$ is defined in Eq. 6

\begin{tabular}{|c|c|c|c|c|}
\hline Nature & 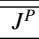 & Mass [MeV] & $\hat{f}_{X}[\mathrm{keV}]$ & $f_{X}(4.5)[\mathrm{keV}]$ \\
\hline \multicolumn{5}{|c|}{$\overline{b \text {-quark channel }}$} \\
\hline \multicolumn{5}{|c|}{ Molecule } \\
\hline $\bar{B}^{*} K$ & $1^{+}$ & $5186 \pm 13$ & $4.48 \pm 0.92$ & $8.02 \pm 1.64$ \\
\hline $\bar{B} K$ & $0^{+}$ & $5195 \pm 15$ & $2.58 \pm 0.55$ & $8.26 \pm 1.76$ \\
\hline $\bar{B}_{s}^{*} \pi$ & $1^{+}$ & $5198 \pm 17$ & $5.32 \pm 0.87$ & $9.51 \pm 1.55$ \\
\hline $\bar{B}_{s} \pi$ & $0^{+}$ & $5202 \pm 24$ & $3.04 \pm 0.54$ & $9.74 \pm 1.74$ \\
\hline \multicolumn{5}{|c|}{ Four-quark $(b u)(\overline{d s})$} \\
\hline$A_{b}$ & $1^{+}$ & $5186 \pm 16$ & $5.05 \pm 1.03$ & $9.04 \pm 1.84$ \\
\hline$S_{b}$ & $0^{+}$ & $5196 \pm 17$ & $2.98 \pm 0.57$ & $9.99 \pm 1.90$ \\
\hline \multicolumn{5}{|c|}{$c$-quark channel } \\
\hline \multicolumn{5}{|c|}{ Molecule } \\
\hline $\bar{D}^{*} K$ & $1^{+}$ & $2395 \pm 44$ & $155 \pm 29$ & $226 \pm 42$ \\
\hline $\bar{D} K$ & $0^{+}$ & $2402 \pm 42$ & $139 \pm 24$ & $254 \pm 48$ \\
\hline $\bar{D}_{s}^{*} \pi$ & $1^{+}$ & $2401 \pm 48$ & $206 \pm 31$ & $277 \pm 41$ \\
\hline $\bar{D}_{s} \pi$ & $0^{+}$ & \pm 36 & \pm 24 & \pm 46 \\
\hline \multicolumn{5}{|c|}{ Four-quark $(\mathrm{cu})(\overline{d s})$} \\
\hline$A_{c}$ & $1^{+}$ & $2400 \pm 46$ & $192 \pm 37$ & $260 \pm 50$ \\
\hline$S_{c}$ & $0^{+}$ & $2395 \pm 67$ & $122 \pm 21$ & $221 \pm 39$ \\
\hline
\end{tabular}

mixed $(\overline{c u}) d s$ and $(\overline{b u}) d s$ exotic hadrons (if any) via eventually their radiative or $\pi+$ hadrons decays.

\section{Conclusion}

We have presented improved predictions of QSSR for the masses and couplings of molecules and fourquarks states at N2LO of PT series and including nonperturbative contributions of condensates up to dimension $d \leqslant 6$. We can see a good convergence of the PT series after including higher order corrections which confirms the veracity of our results. For the charm (resp. bottom) channels, our predictions for molecules and four-quark vector non-strange states are too high compared with the observed $Y_{c}(4140)$ to $Y_{c}$ (4660) (resp. $\left.Y_{b}(9898,10260,10870)\right)$. We can also notice that the SU3 breakings are tiny for the masses ( $\leqslant 10$ (resp. 3)\%) for the charm (resp. bottom) channels but can be large for the couplings $(\leqslant 20 \%)$. This can be understood as in the ratios of sum rules, the corrections tend to cancel out. Our analysis has been done within stability criteria with respect to the LSR variable $\tau$, the QCD continuum threshold $\sqrt{t_{c}}$ and the subtraction constant $\mu$ which have provided successful predictions in different hadronic channels [9, 10]. The optimal values of the masses and couplings have been extracted at the same value of these parameters where the stability appears as an extremum and/or inflection points. We do not include higher dimension condensates contributions (which are not under good control) in our estimate but only consider them as a source of errors. This work is an improvement of all existing previous LO results in the literature from QCD spectral sum rules on XYZ like masses and couplings. We expect that future experimental data and/or lattice results will check our predictions. 


\section{References}

[1] R. Albuquerque, S. Narison, D. Rabetiarivony and G. Randriamanatrika, Int. J. Mod. Phys. A33 (2018), 1850082.

[2] R. Albuquerque, S. Narison, F. Fanomezana, A. Rabemananjara, D. Rabetiarivony and G. Randriamanatrika, Int. J. Mod. Phys. A31 (2016) no. 36, 1650196.

[3] R. Albuquerque, S. Narison, A. Rabemananjara and D. Rabetiarivony, Int. J. Mod. Phys. A31 (2016) no.17, 1650093.

[4] M.A. Shifman, A.I. Vainshtein and V.I. Zakharov, Nucl. Phys. B147 (1979) 385, 448

[5] J.S. Bell and R.A. Bertlmann, Nucl. Phys. B177 (1981) 218; Nucl. Phys. B187 (1981) 285.

[6] R.A. Bertlmann, Acta Phys. Austriaca 53 and references therein.

[7] R.A. Bertlmann and H. Neufeld, Z. Phys. C27 (1985) 437.

[8] S. Narison and E. de Rafael, Phys. Lett. B 522 (2001) 266.

[9] S. Narison, QCD as a theory of hadrons, Cambridge Monogr. Part. Phys. Nucl. Phys. Cosmol. 17 (2002) 1 [hep-ph/0205006].

[10] S. Narison, QCD spectral sum rules, World Sci. Lect. Notes Phys. 26 (1989) 1.

[11] S. Narison, Phys. Rept. 84 (1982) 263; S. Narison, Acta Phys. Pol. B 26 (1995) 687.

[12] E. de Rafael, hep-ph/9802448.

[13] R.D. Matheus, S. Narison, M. Nielsen and J.M. Richard, Phys. Rev. D75 (2007) 014005

[14] R.M. Albuquerque, F. Fanomezana, S. Narison and A. Rabemananjara, Nucl. Phys. Proc. Suppl. 234 (2013) 158-161.

[15] S. Narison, F.S. Navarra, M. Nielsen, Phys. Rev. D83 (2011) 016004.

[16] R. Albuquerque, S. Narison, F. Fanomezana, A. Rabemananjara, D. Rabetiarivony, G. Randriamanatrika, Nucl. Part. Phys. Proc. 282-284 (2017) 83.

[17] F. Fanomezana, S. Narison and A. Rabemananjara, Nucl. Part. Phys. Proc. 258-259 (2015) 156.

[18] C. Becchi, S. Narison, E. de Rafael and F.J. Yndurain, Z. Phys. C8 (1981) 335.

[19] A. Pich and E. de Rafael, Phys. Lett. B158 (1985) 477.

[20] S. Narison, Phys.Rev. D74 (2006) 034013.

[21] S. Narison, Phys. Lett. B605 (2005) 319.

[22] S. Narison, Phys.Lett. B466 (1999) 345.

[23] S. Narison, Phys. Lett. B387 (1996) 162.

[24] S. Narison, Phys. Lett. B337 (1994) 166.

[25] S. Narison, Phys. Lett. $B 358$ (1995) 113; Phys. Lett. $B 322$ (1994) 327.

[26] S. Narison, Phys. Lett. B210 (1988) 238.

[27] R.M. Albuquerque and S. Narison, Phys. Lett. B694 (2010) 217

[28] R.M. Albuquerque, S. Narison and M. Nielsen, Phys. Lett. B684 (2010) 236.

[29] S. Narison, F. Navarra and M. Nielsen, Phys. Rev. D83 (2011) 016004 .

[30] S. Narison, Phys. Lett. B718 (2013) 1321.

[31] S. Peris, B. Phily and E. de Rafael, Phys. Rev. Lett. 86 (2001) 14.

[32] S. Narison, Int. J. Mod. Phys. A33 (2018) no. 10, 1850045.

[33] C. McNeile et al., Phys. Rev. D87 (2013) no.3, 034503.

[34] E.G. Floratos, S. Narison and E. de Rafael, Nucl. Phys. B155 (1979) 155.

[35] S. Narison, Phys. Lett. B673 (2009) 30.

[36] E. Braaten, S. Narison and A. Pich, Nucl. Phys. B373 (1992) 581.

[37] S. Narison and A. Pich, Phys. Lett. B211 (1988) 183.

[38] For reviews, see e.g: S. Bethke, Nucl. Part. Phys. Proc.
282-284 (2017)149; A. Pich, arXiv:1303.2262, [PoSConfinementX,022 (2012)]; G. Salam, arXiv:1712.05165 [hep-ph].

[39] H.G. Dosch and S. Narison, Phys. Lett. B417 (1998) 173.

[40] S. Narison, Phys. Lett. B216 (1989) 191.

[41] S. Narison, Phys. Lett. B738 (2014) 346.

[42] S. Narison, arXiv:hep-ph/0202200 (2002).

[43] S. Narison, Phys. Lett. $\mathbf{B 6 9 3}$ (2010) 559; Erratum ibid 705 (2011) 544

[44] S. Narison, Phys. Lett. B706 (2011) 412.

[45] S. Narison, Phys. Lett. B707 (2012) 259.

[46] PDG, C. Patrignani et al. (Particle Data Group), Chin. Phys. C40, 100001 (2016) and 2017 update.

[47] B.L. Ioffe and K.N. Zyablyuk, Eur. Phys. J. C27 (2003) 229.

[48] B.L. Ioffe, Prog. Part. Nucl. Phys. 56 (2006) 232.

[49] Y. Chung et al., Z. Phys. C25 (1984) 151.

[50] H.G. Dosch, Non-Perturbative Methods (Montpellier 1985) ed. S. Narison, World Scientific (Singapore).

[51] H.G. Dosch, M. Jamin and S. Narison, Phys. Lett.B220 (1989) 251.

[52] B.L. Ioffe, Nucl. Phys. B191 (1981) 591.

[53] A.A.Ovchinnikov and A.A.Pivovarov, Yad. Fiz. 48 (1988) 1135 .

[54] G. Launer, S. Narison and R. Tarrach, Z. Phys. C26 (1984) 433.

[55] S. Narison, Phys. Lett. B300 (1993) 293.

[56] S. Narison, Phys. Lett. B361 (1995) 121.

[57] F.J. Yndurain, Phys. Rept. 320 (1999) 287.

[58] S. Narison, Phys. Lett. B624 (2005) 223.

[59] D.J. Broadhurst, Phys. Lett. B101 (1981) 423.

[60] K.G. Chetyrkin and M. Steinhauser, Phys. Lett. $B 502$ (2001) 104; Eur. Phys. J. C21 (2001) 319.

[61] K.G. Chetyrkin and M. Steinhauser, Eur. Phys. J. C21 (2001) 319 and references therein.

[62] P. Gelhausen et al., Phys. Rev. D88 (2013) 0141015, Erratum: ibid. D89 (2014) 099901, Erratum: ibid. D91 (2015) 099901.

[63] S. Narison and V.I. Zakharov, Phys. Lett. B679 (2009) 355.

[64] K. Chetyrkin, S. Narison and V.I. Zakharov, Nucl. Phys. B550 (1999) 353

[65] S. Narison and V.I. Zakharov, Phys. Lett. B522 (2001) 266

[66] S. Narison and A. Pivovarov, Phys. Lett. B327 (1994) 341

[67] K. Hagiwara, S. Narison and D. Nomura, Phys. Lett. B540 (2002) 233

[68] J-R Zhang and M.-Q. Huang, J. Phys. G37 (2010) 025005.

[69] R. Albuquerque, M.E. Bracco, and M. Nielsen, Phys. lett. B678 (2009) 186.

[70] Z. G. Wang, Z. C. Liu and X. H. Zhang, Eur. Phys. J. C64 (2009) 373

[71] C. F. Qiao and L. Tang, Eur. Phys. J. C74 (2014) 2810.

[72] J-R Zhang and M-Q Huang, Commun. Theor. Phys. 54 (2010) 1075 .

[73] V.M Abazov et al., [D0 Collaboration], Phys. Rev. D89 (2014) 012004 .

[74] R. Aaij et al., [LHCb collaboration], Phys. Rev. Lett. 117 (2016) 152003

[75] B. Aubert et al., [BABAR collaboration], Phys. Rev. Lett. 90 (2003) 242001 